05

\title{
Резонансные изменения микроволнового коэффициента преломления в пластинах иттриевого феррита-граната
}

\author{
(C) Е.А. Кузнецов, ${ }^{1,2}$ А.Б. Ринкевич, ${ }^{1}$ Д.В. Перов ${ }^{1}$ \\ ${ }^{1}$ Институт фризики металлов им. М.Н. Михеева УрО РАН, \\ 620990 Екатеринбург, Россия \\ ${ }^{2}$ Российский государственный профессионально-педагогический университет, \\ 620012 Екатеринбург, Россия \\ e-mail: kuzeag@mail.ru
}

Поступило в Редакцию 24 августа 2018 г.

В окончательной редакции 24 августа 2018 г.

Принято к публикации 19 ноября 2018 г.

\begin{abstract}
Экспериментально и теоретически изучено влияние микроволновых резонансных явлений на комплексный коэффициент преломления в пластинах иттриевого феррита-граната на частотах от 26 до $38 \mathrm{GHz}$. При изменении магнитного поля обнаружены сильные изменения резонансного типа в коэффициентах прохождения и отражения. Установлено, что эти изменения обусловлены как высокой эффективностью взаимодействия электромагнитных волн миллиметрового диапазона с пластинами иттриевого феррита-граната, в частности, при достижении условия ферромагнитного резонанса, так и выполнением условий геометрических резонансов, когда на толщине пластины укладывается целое число половин или целое нечетное число четвертей длины волны. Предложен алгоритм расчета комплексного коэффициента преломления с учетом тензорного характера магнитной проницаемости иттриевого феррита-граната. Получены и проанализированы полевые и частотные зависимости комплексного коэффициента преломления. Проведено сопоставление величин полей геометрических резонансов экстремумам полевых зависимостей модулей коэффициентов отражения и прохождения.
\end{abstract}

DOI: $10.21883 /$ JTF.2019.05.47468.324-18

\section{Введение}

Ферриты нашли широкое применение при производстве СВЧ-устройств диапазона $1-110 \mathrm{GHz}$ : циркуляторов, вентилей, резонаторов, фильтров, фазовращателей благодаря уникальным свойствам - большим значениям удельного сопротивления, высокой магнитной проницаемости и высокой температуре Кюри $[1,2]$.

Первоначально применение в СВЧ-технике нашли ферриты-шпинели. В настоящее время разработчики многих устройств отдают предпочтение ферритам-гранатам - ферритам иттрия и лантаноидам, что обусловлено их наиболее низкими магнитными потерями [3]. Гранаты имеют сложную структуру и общую химическую формулу в виде $\mathrm{R}_{3} \mathrm{Fe}_{5} \mathrm{O}_{12}$, где $\mathrm{R}$ один из трехвалентных ионов редкоземельных элементов (Y, Gd, Tb, Dy, Ho, Er, Sm или Eu) [4]. Эти материалы обладают поликристаллической структурой и производятся на основе керамической технологии. Важнейшей характеристикой их является весьма малая ширина линии ферромагнитного резонанса (ФМР) [5]. Следует отметить, что при экспериментальном определении ширины линии ферромагнитного резонанса $\Delta H$ результат измерений зависит от качества полировки поверхности образца: чем выше класс обработки, тем уже линия ФМР [6]. Хотя ферриты-гранаты являются достаточно хорошо изученным материалом благодаря уникальным магнитным свойствам на СВЧ они еще далеко не исчерпали своих возможностей. В настоящее время проводятся активные исследования таких явлений в тонких пленках железо-иттриевого феррита-граната (ЖИГ) как спиновые волны пар темных солитонов [7] и широкополосный динамический хаос в активном кольце $[8]$.

Однако до настоящего времени не было проведено систематического исследования микроволнового коэффициента преломления ЖИГ. Определить микроволновой коэффициент можно по измерениям коэффициентов прохождения электромагнитных волн через пластину и отражения от нее. Исследование коэффициента преломления на микроволнах приобрело особое значение после того, как было выяснено, что этот коэффициент при определенных условиях может принимать аномальные значения, например, отрицательные или близкие к нулю [9]. Таким образом, весьма актуальной является задача изучения изменения микроволновых коэффициентов прохождения и отражения под действием постоянного магнитного поля и влияние этих изменений на поведение комплексного коэффициента преломления в условиях не только ФМР, но и геометрических резонансов, когда на толщине пластины укладывается целое нечетное число четверть или целое число полуволн. Ожидается, что совместное действие этих факторов может привести к экстраординарным изменениям коэффициентов прохождения и отражения. 


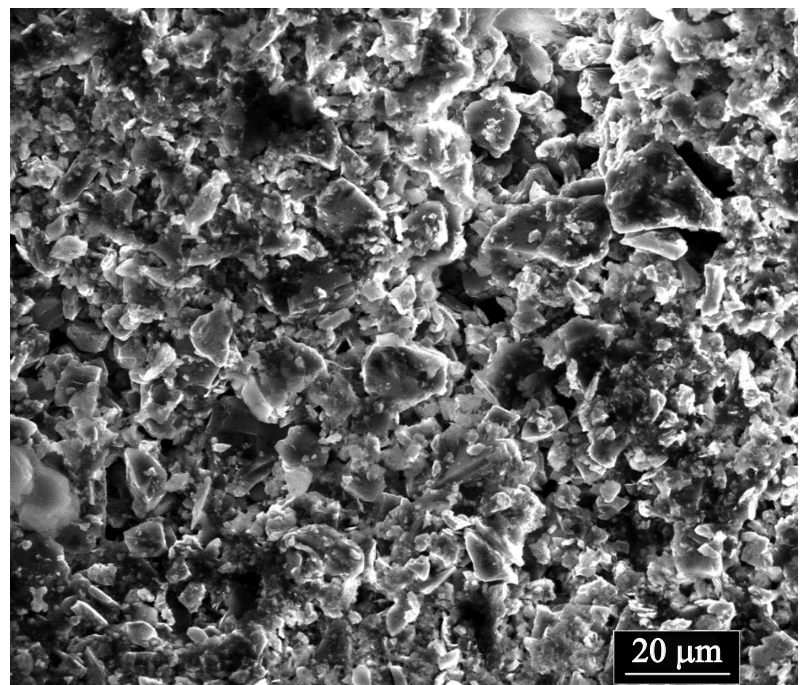

Pис. 1. Электронно-микроскопическое изображение образца № 1 толщиной $1.1 \mathrm{~mm}$.

\section{Объекты исследования}

В настоящей работе исследовались два поликристаллических образца иттриевого феррита-граната $\mathrm{Y}_{3} \mathrm{Fe}_{5} \mathrm{O}_{12}$, синтезированные по золь-гелевой технологии из нитрата иттрия, нитрата железа и водного раствора лимонной кислоты в АО НИИ „Феррит-Домен“ из г. Санкт-Петербурга. Образец № 1 имеет полированную поверхность с шероховатостью $\sim(1-2) \mu \mathrm{m}$ и толщину $d=1.1 \mathrm{~mm}$, тогда как образец № 2 имеет шероховатую поверхность с максимальной шероховатостью около $100 \mu \mathrm{m}$ и толщиной $d=2.0 \mathrm{~mm}$. Микроструктурные исследования выполнены в Центре коллективного пользования ИФМ УрО РАН на сканирующем электронном микроскопе Quanta 200 с ускоряющим напряжением до $30 \mathrm{kV}$. Микроскоп оборудован системой Pegasus, позволяющей проводить исследования дифракции на обратном рассеянии электронов (EBSD). Микроструктура образца № 1, полученного при обратном рассеянии электронов с усилением 2000, показана на рис. 1. Средний размер зерна - от $8-10 \mu \mathrm{m}$. Химический анализ образцов выполнен с помощью системы EDAX. Для образца № 1 был получен следующий состав: $\mathrm{O}-$ $54.6 \%, \mathrm{Fe}-29.3 \%, \mathrm{Y}-16.1 \%$. Почти идентичный результат был получен для образца № 2: О - 53.8\%, $\mathrm{Fe}-27.3 \%, \mathrm{Y}-18.9 \%$. Все эти результаты приблизительно соответствуют номинальному химическому составу $\mathrm{Y}_{3} \mathrm{Fe}_{5} \mathrm{O}_{12},(\mathrm{O}-60.0 \%, \mathrm{Fe}-25.0 \%, \mathrm{Y}-15 \%)$.

\section{Методика микроволновых измерений и их результаты}

Измерения были выполнены в полосе частот 26-38 GHz методом, развитым ранее и описанным в [10]. Образец размещен в поперечном сечении пря- моугольного волновода и полностью его перекрывает. Волновод имеет размеры $7.2 \times 3.4 \mathrm{~mm}$ и работает на основной моде $\mathrm{H}_{10}$. Для разделения прямых и обратных волн использованы направленные ответвители. Модули коэффициентов прохождения $D$ и отражения $R$ и их зависимости от внешнего магнитного поля измерены с помощью скалярного анализатора цепей. Их относительные изменения определены как $d_{m}=[|D(H)|-|D(0)|] /|D(0)|$, где $|D(H)|-$ модуль коэффициента прохождения в магнитном поле $H$, и $r_{m}=[|R(H)|-|R(0)|] /|R(0)|$, где $|R(H)|$ - модуль коэффициента отражения в постоянном магнитном поле $H$. Постоянное магнитное поле $H$ лежит в плоскости пластины и направлено перпендикулярно к сверхвысокочастотному магнитному полю $H_{\sim}, H \perp H_{\sim}$. Все микроволновые измерения выполнены при комнатной температуре.

В измерениях были получены аномально большие до $+950 \%$ - относительные изменения в магнитном поле модулей коэффициентов прохождения $d_{m}$ и отражения $r_{m}$. На рис. 2 показаны полевые зависимости коэффициентов отражения, прохождения и диссипации для образца № 2 на частоте $29 \mathrm{GHz}$. Диссипация $\Delta$ рассчитывается из измеренных коэффициентов $D$ и $R$ :

$$
\Delta=1-|D|^{2}-|R|^{2} .
$$

Параметр $\Delta$ выражает долю мощности, поглощенную и рассеянную в образце. При увеличении магнитного поля от 0 до $8.4 \mathrm{kOe}$ коэффициент отражения достигает минимума в 0.046, коэффициент прохождения максимума 0.637 при локальном максимуме диссипации 0.317. В полях 9.6-9.8 kОе диссипация становится минимальной, $\Delta=0.013$, как и коэффициент прохождения $D=0.054$, а коэффициент отражения оказывается максимальным $-R=0.958$. И, наконец, внутри интервала полей $10.4-10.8 \mathrm{kOe}$ диссипация достигает максимума 0.697, коэффициент прохождения - минимума $D=0.003$ при локальном минимуме отражения

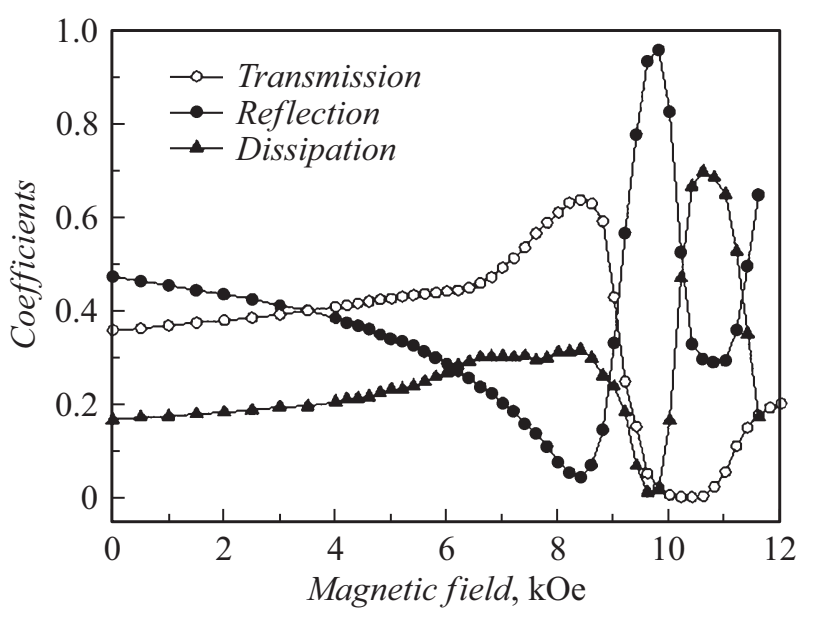

Рис. 2. Прохождение микроволн через пластину YIG образца № 2: сравнение полевых зависимостей коэффициентов прохождения, отражения и диссипации на частоте $29 \mathrm{GHz}$. 
0.290; этот интервал соответствует условию ФМР. Итак, в интервале полей 0-12 kОе коэффициент отражения меняется в 21 раз, диссипации в 52 раза, а коэффициент прохождения - почти в 240 раз.

\section{Обсуждение}

Вычисление комплексного коэффициента преломления было выполнено с использованием материальных констант: диэлектрической проницаемости $\varepsilon$ и эффективной динамической магнитной проницаемости $\mu_{\text {eff }}$. Он определяется следующим образом:

$$
n=n^{\prime}-i n^{\prime \prime}=\sqrt{\varepsilon \mu_{\mathrm{eff}}},
$$

где $n^{\prime}$ - коэффициент преломления и $n^{\prime \prime}-$ коэффициент поглощения.

Для нахождения комплексной диэлектрической проницаемости $\varepsilon$ использовался алгоритм ее восстановления с помощью вычисления разности между модулями теоретических и экспериментальных частотных зависимостей коэффициентов прохождения и отражения, описанный
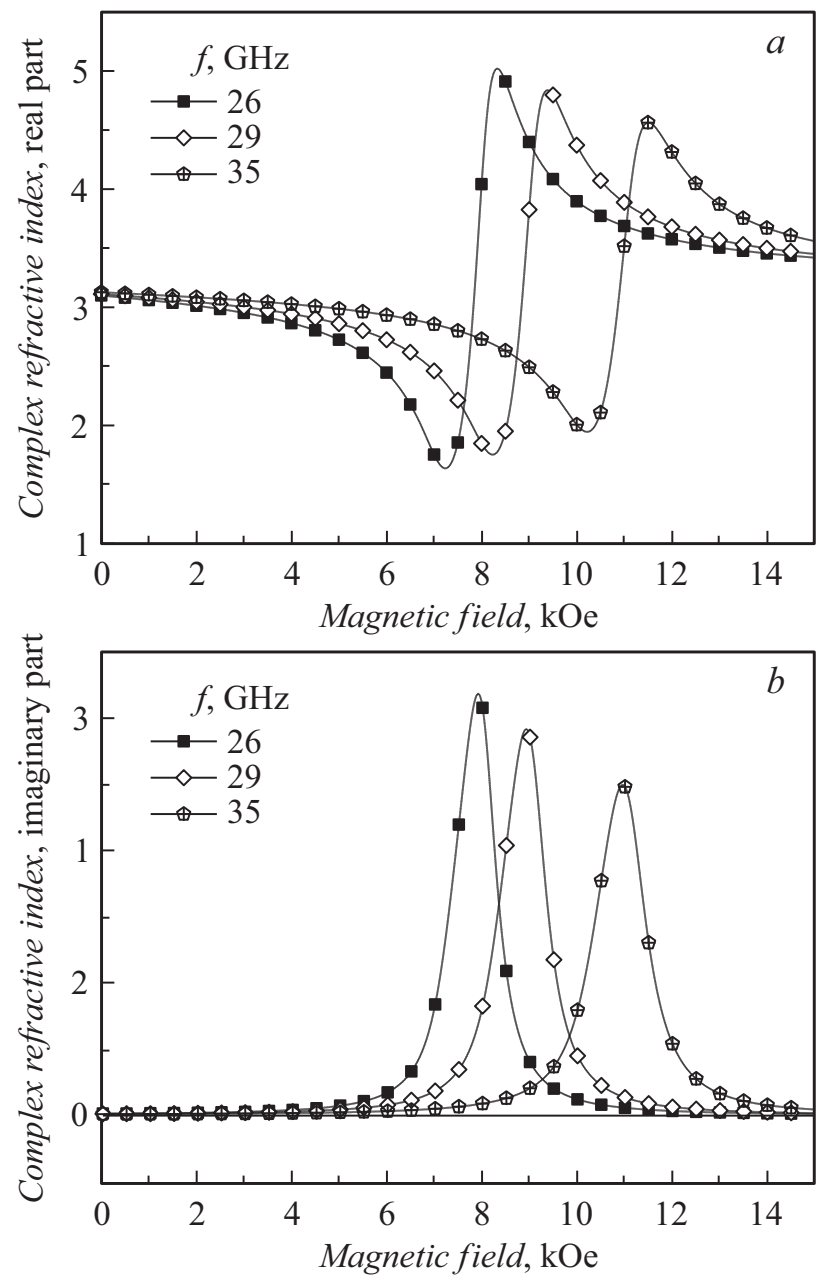

Рис. 3. Полевые зависимости комплексного коэффициента преломления микроволн в образце № 2: $a$ - действительная часть, $b-$ мнимая часть.
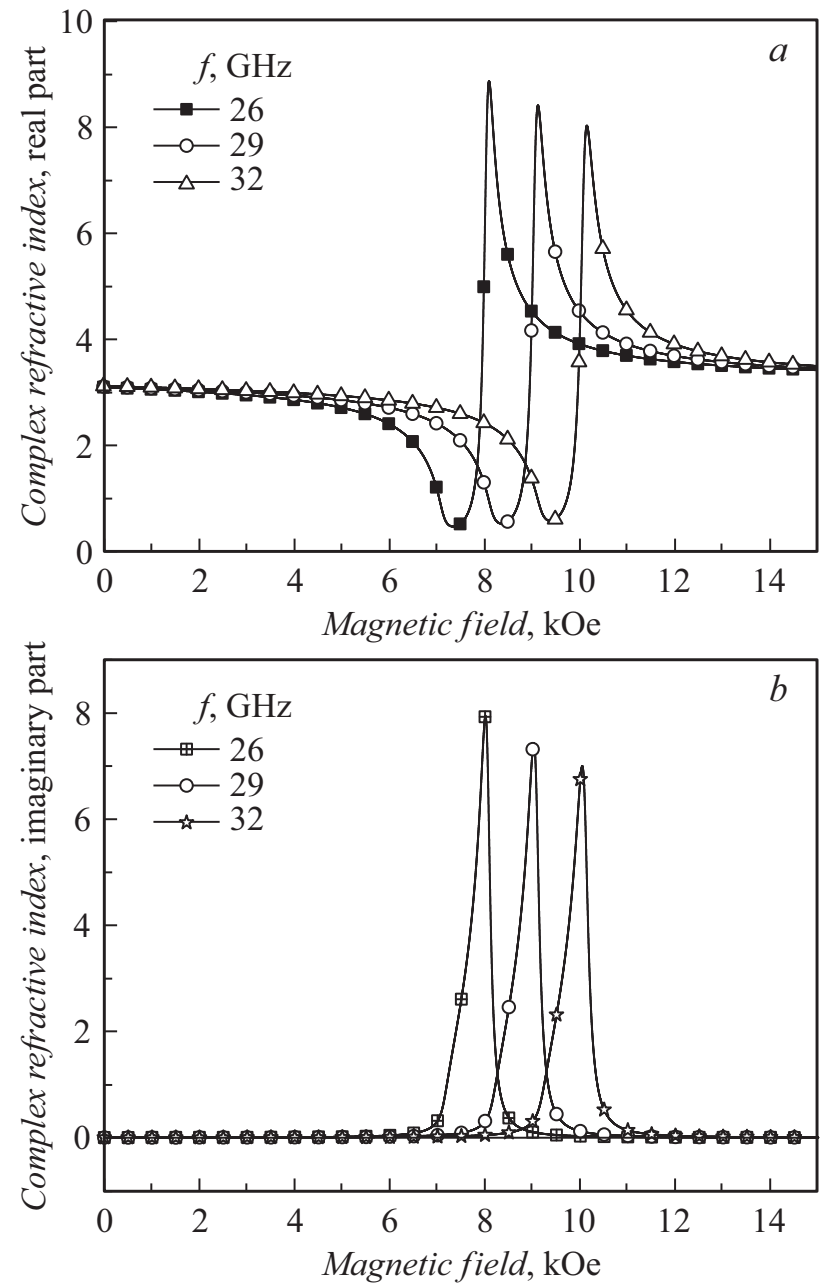

Рис. 4. Полевые зависимости комплексного коэффициента преломления микроволн в образце № 1: $a$ - действительная часть, $b-$ мнимая часть.

в [10]. Затем применялся метод наименьших квадратов для минимизации этой разности. Было получено, что вещественная часть комплексной диэлектрической проницаемости $\varepsilon^{\prime}=10.8$, а мнимая часть $\varepsilon^{\prime \prime}$ много меньше, чем вещественная, $\varepsilon^{\prime \prime} \ll \varepsilon^{\prime}$. Мы далее полагаем значение $\varepsilon^{\prime}$ постоянным, не зависящим от магнитного поля.

Магнитная проницаемость ЖИГ в магнитном поле есть величина тензорная. В наших экспериментальных условиях волновой вектор перпендикулярен вектору напряженности постоянного магнитного поля и плоскости пластины образца, $\mathbf{q} \perp \mathbf{H}_{0}$, и эффективная магнитная

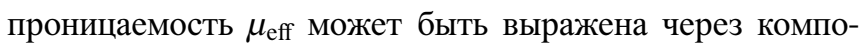
ненты тензора динамической проницаемости [5]:

$$
\mu_{\mathrm{eff}}=\mu-\frac{\mu_{a}^{2}}{\mu} .
$$

В формуле (2) $\mu$ - диагональные и $\mu_{a}$ - недиагональные компоненты тензора магнитной проницаемости. 

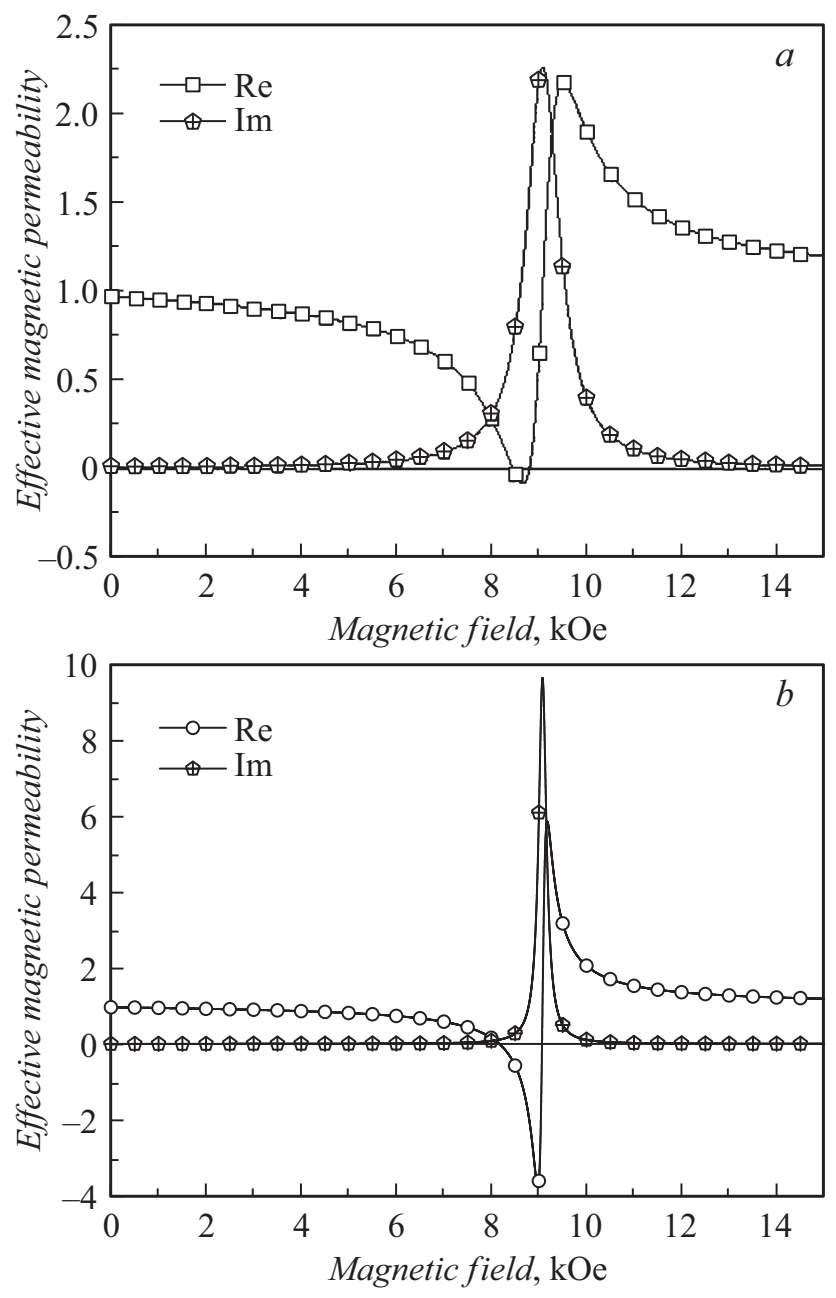

Рис. 5. Полевые зависимости комплексной эффективной магнитной проницаемости на частоте $29 \mathrm{GHz}: a-$ образца № 2 , $b$ - образца № 1 .

Чтобы вычислить эффективную магнитную проницаемость, нужны частотные зависимости и зависимости компонент $\mu$ и $\mu_{a}$ от магнитного поля, выраженные в явной форме. Для оценки достаточно предположить обычную лоренцеву форму зависимостей.

Далее воспользуемся выражением для тензора динамической магнитной проницаемости [5]:

$$
\overleftrightarrow{\mu}=\left(\begin{array}{ccc}
\mu & i \mu_{a} & 0 \\
-i \mu_{a} & \mu & 0 \\
0 & 0 & \mu_{\|}
\end{array}\right)
$$

где $\mu=1+4 \pi \chi, \mu=4 \pi \chi_{a}$ и $\mu_{\|}=1+4 \pi \chi_{\|}$. Динамическая магнитная восприимчивость здесь выражена в виде

$$
\begin{aligned}
\chi= & \chi^{\prime}-i \chi^{\prime \prime}=\gamma M_{0} \\
& \times \frac{\omega_{H}\left[\omega_{H}^{2}-\left(1-\alpha^{2}\right) \omega^{2}\right]-i \alpha \omega\left[\omega_{H}^{2}+\left(1+\alpha^{2}\right) \omega^{2}\right]}{L},
\end{aligned}
$$

$$
\begin{gathered}
\chi_{a}=\chi_{a}^{\prime}-i \chi_{a}^{\prime \prime}=\gamma M_{0} \omega \frac{\left[\omega_{H}^{2}-\left(1+\alpha^{2}\right) \omega^{2}\right]-i 2 \alpha \omega \omega_{H}}{L}, \\
\chi_{\|}=-\frac{i \alpha \gamma M_{0}}{\omega-i \alpha \omega_{H}}
\end{gathered}
$$

где $L=\left[\omega_{H}^{2}-\left(1+\alpha^{2}\right) \omega^{2}\right]^{2}+4 \alpha^{2} \omega^{2} \omega_{H}^{2}, \gamma=(g|e|) /(2 m c)-$ гиромагнитное отношение, $g$ - коэффициент спектроскопического расщепления, $e-$ электрический заряд электрона, $m$ - эффективная масса электрона, $c$ скорость распространения электромагнитной волны в вакууме, $\omega_{H}=\gamma H_{0}, \alpha-$ безразмерный параметр затухания в уравнении Ландау-Лифшица-Гильберта и $M_{0}$ - намагниченность насыщения [5]. Ширина линии ферромагнитного резонанса $\Delta H$ определялась из эксперимента. Для граната $\mathrm{Y}_{3} \mathrm{Fe}_{5} \mathrm{O}_{12}$ намагниченность насыщения $4 \pi M_{0}=1750 \mathrm{G}$. Параметр затухания определялся по ширине пика коэффициента диссипации и составил для образца № $2 \alpha=0.043$, а для образца № $1 \alpha=0.01$.

На рис. 3 и 4 приведены полевые зависимости для комплексного коэффициента преломления $n$, а на рис. 5 - полевые зависимости для комплексной эффективной магнитной проницаемости $\mu_{\text {eff. Зависимости }}$ действительных частей показателя преломления имеют вид резонансных кривых, положение которых по оси абсцисс смещается в область более сильных полей при увеличении частоты. Зависимости мнимых частей показателя преломления имеют вид резонансных максимумов. Разумеется, полученные резонансные зависимости коэффициента преломления - это результат изменения динамической магнитной проницаемости при ФМР. Поскольку, как уже упоминалось, образцы различаются не только толщиной, но и качеством обработки поверхности, ширина пика мнимой части эффективной магнитной проницаемости и соответственно коэффициента преломления для образца № 1 меньше, а высота его больше. Действительные части $\mu_{\text {eff }}$ и $n$ также сильнее изменяются у образца № 1 .

Весьма интересным результатом является то, что у образца № 1 в диапазоне магнитных полей, меньших поля ФМР, вещественная часть комплексного коэффициента преломления становится меньше единицы, $n^{\prime}<1$. Эти аномальные значения показателя преломления получились в результате сильных изменений $\mu_{\text {eff. }}$ Возникает вопрос, не подобен ли ЖИГ при условии $n^{\prime}<1$ так называемому ENZ-материалу [11]? В обоих случаях действительная часть показателя преломления положительна, но меньше единицы. Для ENZ-материала это необычное значение показателя преломления достигается за счет малой действительной части диэлектрической проницаемости. В нашем случае диэлектрическая проницаемость велика $\left|\varepsilon^{\prime}\right| \gg 1$, а необычное значение $n^{\prime}$ достигается благодаря изменениям магнитной проницаемости, причем в условиях сильного поглощения. Из-за сильного поглощения между нашим случаем и ENZ-материалами нет прямой аналогии, подобно работе [12]. Отметим, что в нашем случае не следует 

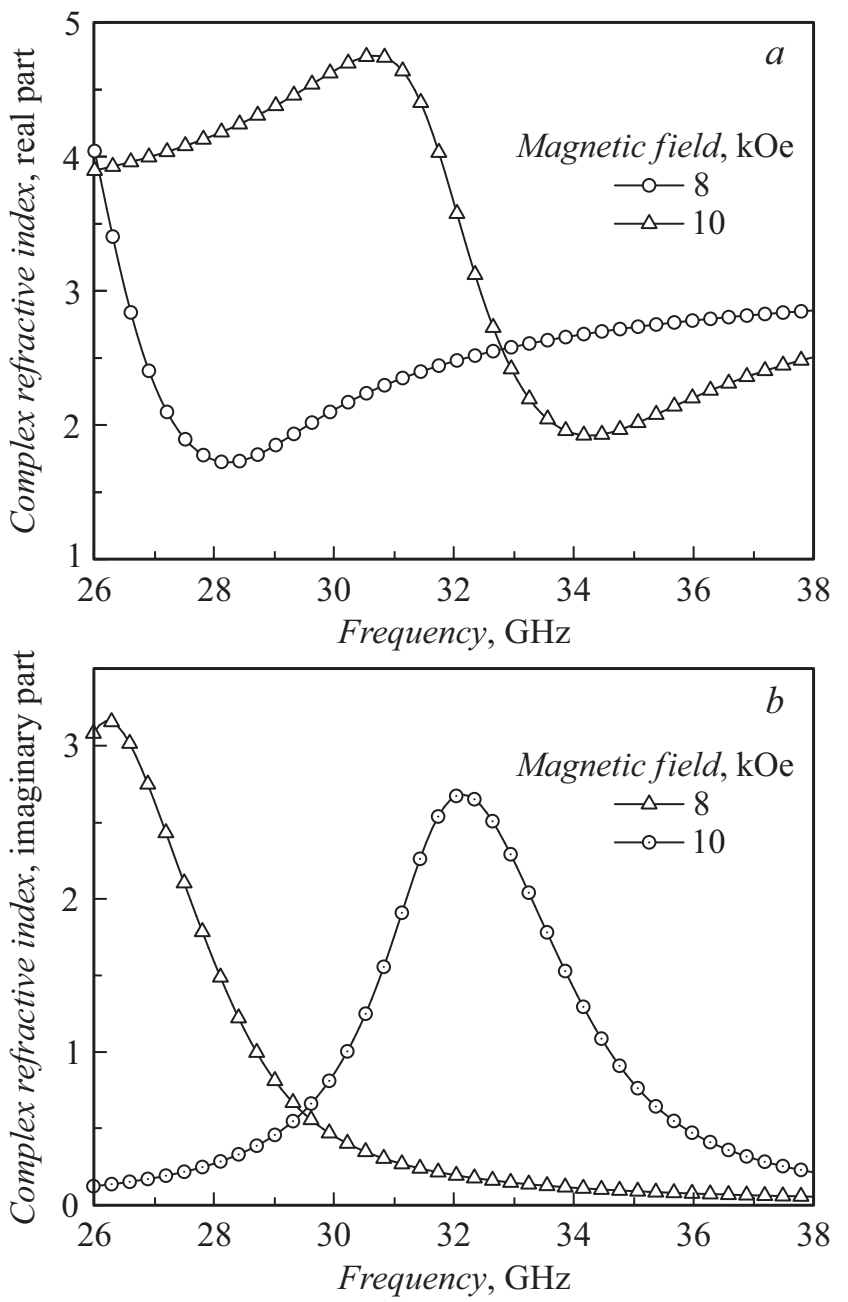

Рис. 6. Частотные зависимости комплексного коэффициента преломления микроволн в образце № 2 в магнитных полях 8 и $10 \mathrm{kOe:} a-$ действительная часть, $b-$ мнимая часть.

ожидать, что изменениями параметров можно добиться отрицательных значений показателя преломления $n^{\prime}<0$. Действительно, отрицательный знак у показателя преломления получается, если действительные части обеих материальных постоянных - и диэлектрической, и магнитной проницаемостей - отрицательны, т.е. в „double left-handed media“ [9]. В нашем случае диэлектрическая проницаемость положительна.

На рис. 6 приведены частотные зависимости $n$, измеренные в разных полях и имеющие немонотонный характер изменения. Немонотонные изменения коэффициентов отражения и прохождения обусловлены как высокой эффективностью взаимодействия электромагнитных волн миллиметрового диапазона с пластинами иттриевого феррита-граната, особенно в условиях ФМР, так и выполнением условий геометрических резонансов, когда на толщине пластины укладывается целое нечетное число четверть и целое число полуволн. Выполнение условий геометрических резонансов соответствует установлению стоячих волн в пластине.
Проанализируем особенности поведения этих коэффициентов для образца № 2 (рис. 7, $a, b)$. Налицо подобие качественного характера экспериментальных и расчетных зависимостей. Количественного согласия нет из-за того, что рассчитанное и экспериментально измеренное поля ФМР отличаются друг от друга, возможно, из-за пористости в образце и из-за влияния размагничивающего фактора образца. На рисунках отмечено положение геометрических резонансов, когда $d=\lambda / 2$ и $3 \lambda / 4$. Под величиной $\lambda$ мы понимаем длину электромагнитной волны в волноводе при выбранной частоте и данном значении магнитного поля. Наличие двух полей, при которых выполняется условие резонанса $d=\lambda / 2$, вызвано немонотонным изменением действительной части комплексной магнитной проницаемости $\mu^{\prime}$ (рис. 5, $a$ ).

Максимум коэффициента прохождения $D$ и минимум коэффициента отражения $R$ вблизи поля $H=6.2 \mathrm{kOe}$ обусловлены геометрическим резонансом $d=\lambda / 2$, при
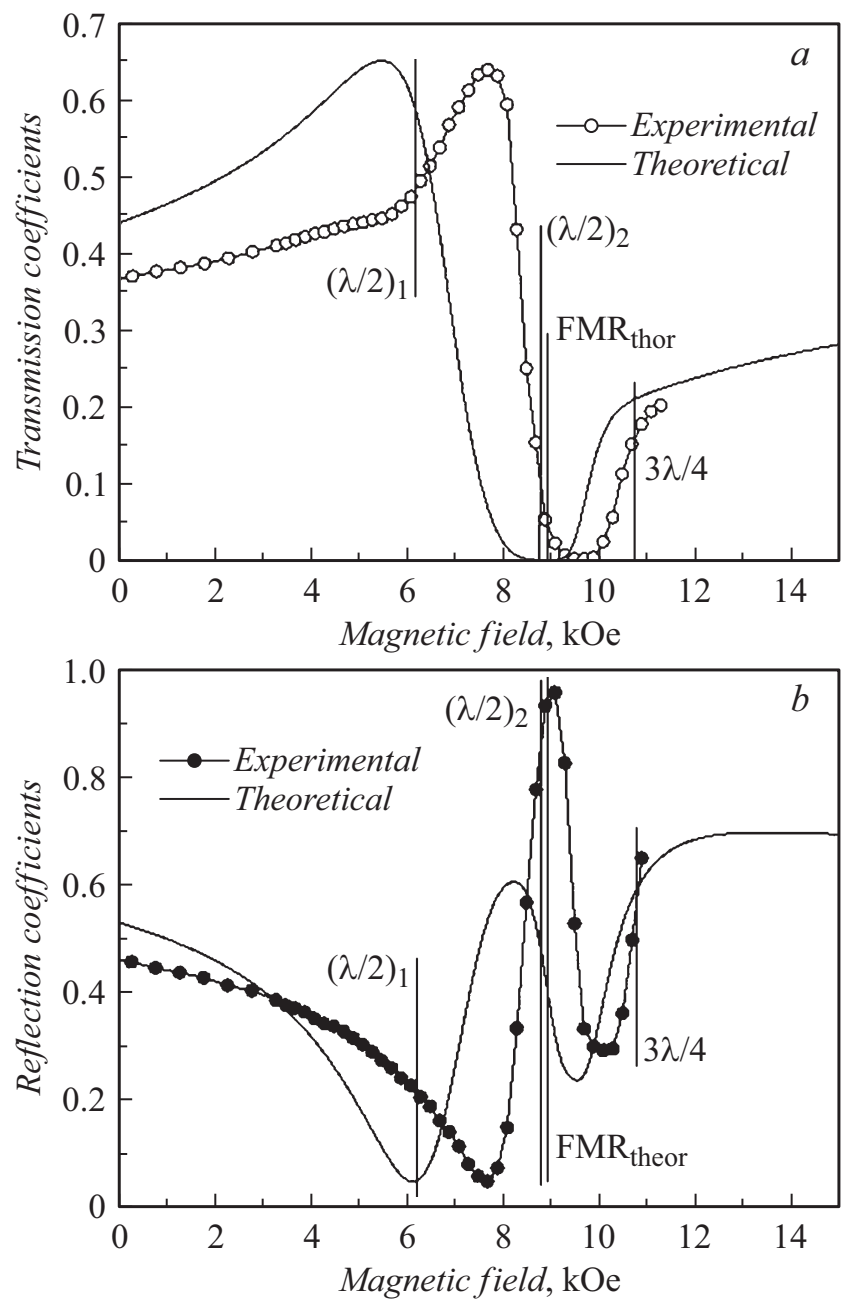

Рис. 7. Прохождение микроволн через пластину YIG образца № 2: сравнение экспериментальных и теоретических полевых зависимостей коэффициентов прохождения (a) и отражения $(b)$ на частоте $29 \mathrm{GHz}$ с указанием теоретических значений полей геометрических резонансов и поля ФМР. 
этом модуль отношения входных импедансов волновода и пластины ЖИГ $\xi=\left|Z_{\text {in1 }} / Z_{\text {in2 }}\right| \approx 1.54$.

При $H \approx 8.8 \mathrm{kOe}$ еще раз выполняется условие резонанса $d=\lambda / 2$. При этом условии обычно достигается большая величина коэффициента прохождения [13]. Однако у нас из-за немонотонного изменения $\mu^{\prime}$ велико отношение импедансов $\xi \approx 3.76$ и получается довольно большое отражение $R \approx 0.48$ (мы сравниваем коэффициенты в условиях геометрических резонансов с расчетной кривой).

В области ФМР при $H \approx 9.1 \mathrm{kOе}$ видны минимумы $D$ и $R$ из-за большого поглощения. Сравнение опять ведется с расчетной зависимостью. Из рис. 5, $a$ видно, что $\mu_{\mathrm{eff}}^{\prime \prime}$ в этом поле достигает максимума.

В поле $H \approx 10.8 \mathrm{kOe} \mathrm{выполняется} \mathrm{условие} d=3 \lambda / 4$. Это условие обычно соответствует минимуму отражения [13]. В нашем случае из-за изменения эффективной магнитной проницаемости отношение входных импедансов достигает большой величины $\xi \approx 8-8.75$. Поэтому неудивительно, что коэффициент отражения велик, $R \approx 0.6-0.7$.

\section{Заключение}

Экспериментально и теоретически исследовано влияние микроволновых резонансных явлений на комплексный коэффициент преломления в пластинах иттриевого феррита-граната на частотах от 26 до $38 \mathrm{GHz}$ двух образцов $\mathrm{Y}_{3} \mathrm{Fe}_{5} \mathrm{O}_{12}$.

Разработан алгоритм расчета комплексного коэффициента преломления $n$ при падении электромагнитной волны на пластину ферромагнитного диэлектрика, помещенного в магнитное поле, с учетом тензорного характера магнитной проницаемости.

Получены и проанализированы полевые и частотные зависимости комплексного коэффициента преломления. Показано, что большие изменения коэффициентов отражения и прохождения приводят к большим изменениям комплексного коэффициента преломления. Физическими причинами его больших изменений являются ферромагнитный резонанс и выполнение условий геометрических резонансов $d=\lambda / 2$ и $d=3 \lambda / 4$.

Работа выполнена при поддержке гранта РНФ 17-12-01002 „Показатель преломления неоднородных сред в магнитном поле и неоднородность микроволнового поля“'. Электронно-микроскопические исследования выполнены в Центре коллективного пользования ИФМ УpO РАH.

\section{Список литературы}

[1] Özgür Ü., Alivov Y., Morkoç H. // J. Mater. Sci.: Materials in Electronics. 2009. Vol. 20. N 9. P. 789-834.

[2] Harris V.G. // IEEE Trans. Mag. 2012. Vol. 48. P. 1075-1104.
[3] Мартынов М.И., Никитин А.А., Устинов А.Б., Калиникос Б.А. Исследование спин-волнового согласованного фильтра на пленке железо-иттриевого граната. Всероссийская научно-техническая конф. „Электроника и микроэлектроника СВЧ“. СПб., СПБГЭТУ, 1-4 июня, 2015. C. $130-134$.

[4] Яковлев Ю.М., Генделев С.Ш. Монокристаллы ферритов в радиоэлектронике / Под ред. Г.А. Матвеева. М.: Советское радио, 1975. $360 \mathrm{c}$.

[5] Гуревич А.Г., Мелков Г.А. Магнитные колебания и волны. М.: Физматлит, 1994. 464 с.

[6] Устинов А., Кочемасов В., Хасьянова Е. // Электроника. 2015. № 8 (00148). C. 86-92.

[7] Wang Z., Cherkasskii M., Kalinikos B.A., Wu M. // Phys. Rev. B. 2015. 91. article 174418.

[8] Kondrashov A., Ustinov A., Cherkasskii M., Kalinikos B.A., Demokritov S.O. Broadband Dynamical Chaos in Active Ring Based on Metalized YIG Film // 8th Joint European Magnetics Symposia, 21-26 August 2016, Glasgow UK.

[9] Electromagnetic Metamaterials: Physics and Engineering Explorations / Ed. by Nader Engheta, R.W. Ziolkowski, WileyIEEE Press, 2006. 440 p.

[10] Rinkevich A.B., Samoilovich M.I., Klescheva S.M., Perov D.V., Burkhanov A.M., Kuznetsov E.A. // IEEE Transactions on Nanotechnology. 2014. Vol. 13. N 1. P. 3-9.

[11] Silveirinha M.G., Engheta N. // Phys. Rev. Lett. 2006. Vol. 97. P. 157403 (1-4).

[12] Rinkevich A.B., Perov D.V., Samoilovich M.I., Klescheva S.M. // Metamaterials. 2012. Vol. 6. N 1-2. P. 27-36.

[13] Бреховских Л.М. Волны в слоистых средах. М.: Изд-во АН CCCP, 1957. $504 \mathrm{c}$. 